\title{
Lacunary Interpolation Using Quartic B-Spline
}

\author{
${ }^{1}$ Karwan Hama Faraj Jwamer and ${ }^{2}$ Bzhar Jamal \\ ${ }^{1,2}$ University of Sulaimani, College of Science, Department of Mathematics, Kurdistan Region, Sulaimani, Iraq
}

karwan.jwamer@univsul.edu.iq

\begin{abstract}
In this paper, a quadratic B-Spline has been constructed that is an approximate solution to a function with very limited given lacunary data and approximate boundary conditions. Then the error bound for the B-spline is found. Further this construction is used to solve boundary value problem.
\end{abstract}

Keywords: B-spline, error bounds, boundary value problems, approximate solution.

2000 MSC No: 65D05, 65D07.

\section{Introduction}

Boundary value problem (BVP) is a differential equation together with some conditions imposed at different points. The BVPs have been applied in mathematics, engineering and various fields of sciences. The rapid increasing of their applications has led to formulating and upgrading several existing methods and new approaches [1].

Quartic B-spline is a piecewise polynomial of degree four satisfying third order parametric continuity [2].

In this paper, quartic B-spline is manipulated to approximate the solution of a BVP with lacunary data given on it and the boundary conditions are approximation, means that it satisfies conditions (1)(2), see below. By presuming the B-spline to be the solution for this problem, an undetermined system of linear equations of order $(n+4) \times(n+4)$ with $n$ being the number of uniform subintervals is built. Adding three approximate boundary conditions into this system gives a square system of $(n+4) \times(n+4)$ that have a unique solution for this problem.

This method can make use of the problem's equation to construct an error equation. Minimization of the error equation would give the value of the variable that produces the best approximation of the solution.

\section{Lacunary Interpolations on Boundary Value Problem using B-spline of Degree four. \\ In this section, we try to find an approximate solution of a function $f(x)$ having very limited lacunary data on it, the function has the following interpolation conditions;}




$$
f\left(x_{i}\right)=y_{i} \quad, i=0,1,2, \ldots, n
$$

With the following lacunary boundary conditions

$$
\begin{aligned}
& f^{\prime}\left(x_{0}\right)=y_{0}^{\prime} \\
& f^{\prime}\left(x_{n}\right)=y_{n}^{\prime} \\
& f^{\prime \prime}\left(x_{0}\right)=y_{0}^{\prime \prime}
\end{aligned}
$$

The B-spline is non-zero at five knots, we can find the value of $B_{i, 4}$ at the nodal points by

\begin{tabular}{|c|c|c|c|c|c|c|}
\hline & $x_{i-2}$ & $x_{i-1}$ & $x_{i}$ & $x_{i+1}$ & $x_{i+2}$ & $x_{i+3}$ \\
\hline$B_{i, 4}(x)$ & 0 & 1 & 11 & 11 & 1 & 0 \\
\hline$B_{i, 4}^{\prime}(x)$ & 0 & $4 / h$ & $12 / h$ & $-12 / h$ & $-4 / h$ & 0 \\
\hline$B^{\prime \prime}{ }_{i, 4}(x)$ & 0 & $12 / h^{2}$ & $-12 / h^{2}$ & $-12 / h^{2}$ & $12 / h^{2}$ & 0 \\
\hline$B^{\prime \prime \prime}{ }_{i, 4}(x)$ & 0 & $24 / h^{3}$ & $-72 / h^{3}$ & $72 / h^{3}$ & $-24 / h^{3}$ & 0 \\
\hline
\end{tabular}
differentiating it with respect to the value $B_{i, 4}(\mathrm{x})$ and its first three derivatives at the nodal points can be tabulated as in table 1.

Table 1: Values of $B_{i, 4}(\mathrm{x})$ and its first three derivatives at the nodal points

From the definition of B-Spline, the solution of Eq.1 using Quartic B-spline is approximated by;

$$
S(x)=\sum_{j=i-2}^{i+2} c_{j} B_{j}(x) \quad, \quad i=0,1,2, \ldots, n
$$

then

$S^{\prime}(x)=\sum_{j=i-2}^{i+2} c_{j} B^{\prime}{ }_{j}(x) \quad, \quad i=0,1,2, \ldots, n$

and

$$
S^{\prime \prime}(x)=\sum_{j=i-2}^{i+2} c_{j} B^{\prime \prime}{ }_{j}(x) \quad, \quad i=0,1,2, \ldots, n
$$

Now without loss of generality, we can re-write Eq. 3 as below,

$S(x)=c_{i-2} B_{i-2}\left(x_{i}\right)+c_{i-1} B_{i-1}\left(x_{i}\right)+c_{i} B_{i}\left(x_{i}\right)+c_{i+1} B_{i+1}\left(x_{i}\right)+c_{i+2} B_{i+2}\left(x_{i}\right)$

where $i=0,1,2, \ldots, n$ and all other $B_{i+k}$ 's are zero, $k=-4,-3,3,4$

By shifting the B spline to the right side by $k^{\prime} s$ step, mathematically meaning;

$B_{i-k}\left(x_{i}\right)=B_{i}\left(x_{i+k}\right)$ for all, then Eq. 3 can be re-written as follows

$$
\begin{aligned}
& S(x)=c_{i-2} B_{i}\left(x_{i+2}\right)+c_{i-1} B_{i}\left(x_{i+1}\right)+c_{i} B_{i}\left(x_{i}\right)+c_{i+1} B_{i}\left(x_{i-1}\right)+c_{i+2} B_{i}\left(x_{i-2}\right), i= \\
& 0,1,2, \ldots, n
\end{aligned}
$$


Doing the same steps on first and second derivatives of the B-spline in Eqs.4 and 5 respectively, gives

$$
\begin{gathered}
S^{\prime}(x)=c_{i-2} B^{\prime}{ }_{i}\left(x_{i+2}\right)+c_{i-1} B^{\prime}{ }_{i}\left(x_{i+1}\right)+c_{i} B^{\prime}{ }_{i}\left(x_{i}\right)+c_{i+1} B^{\prime}{ }_{i}\left(x_{i-1}\right)+c_{i+2} B^{\prime}{ }_{i}\left(x_{i-2}\right), \\
i=0,1,2, \ldots, n .
\end{gathered}
$$

and

$$
\begin{gathered}
S^{\prime \prime}(x)=c_{i-2} B^{\prime \prime}{ }_{i}\left(x_{i+2}\right)+c_{i-1} B^{\prime \prime}{ }_{i}\left(x_{i+1}\right)+c_{i} B^{\prime \prime}{ }_{i}\left(x_{i}\right)+c_{i+1} B^{\prime \prime}{ }_{i}\left(x_{i-1}\right)+c_{i+2} B^{\prime \prime}{ }_{i}\left(x_{i-2}\right), \\
i=0,1,2, \ldots, n .
\end{gathered}
$$

From the lacunary conditions and by substituting the values of $B_{i, 4}(x)$ at the knots using table 1 , the following equations are formulated;

$$
\begin{aligned}
& f\left(x_{0}\right) \cong S\left(x_{0}\right)=c_{-2}+11 c_{-1}+11 c_{0}+c_{1} \\
& f\left(x_{1}\right) \cong S\left(x_{1}\right)=c_{-1}+11 c_{0}+11 c_{1}+c_{2} \\
& f\left(x_{2}\right) \cong S\left(x_{2}\right)=c_{0}+11 c_{1}+11 c_{2}+c_{3} \\
& \cdot \\
& f\left(x_{n}\right) \cong S\left(x_{n}\right)=c_{n-2}+11 c_{n-1}+11 c_{n}+c_{n+1}
\end{aligned}
$$

From Eq.9, there are $n+4$ unknowns to be founded and $n+1$ equations, so we need to write three more equations which are the lacunary boundary conditions, to have;

$$
\begin{aligned}
& h f^{\prime}\left(x_{0}\right) \cong h S^{\prime}\left(x_{0}\right)=-4 c_{-2}-12 c_{-1}+12 c_{0}+4 c_{1} \\
& h f^{\prime}\left(x_{n}\right) \cong h S^{\prime}\left(x_{n}\right)=-4 c_{n-2}-12 c_{n-1}+12 c_{n}+4 c_{n+1} \\
& h^{2} f^{\prime \prime}\left(x_{0}\right) \cong h^{2} S^{\prime \prime}\left(x_{0}\right)=12 c_{-2}-12 c_{-1}-12 c_{0}+12 c_{1}
\end{aligned}
$$

Eqs. 9 and 10 forms a square system of $(n+4) x(n+4)$ system represented in the matrix form as follows 


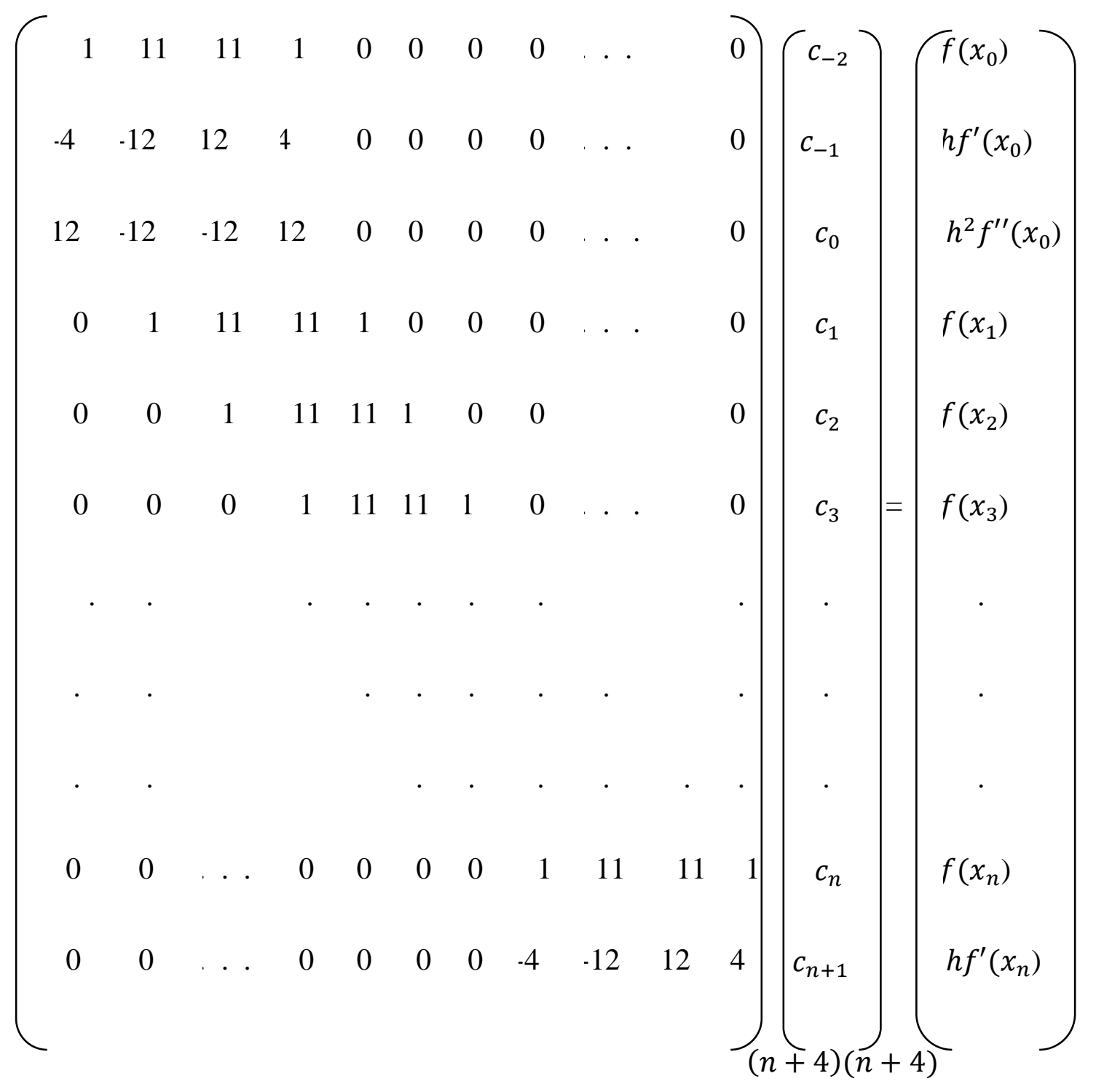

Above Matrix is invertible, to show this we can use the principal of mathematical induction, this yields that above

system has a unique solution.

With this, the construction of a fourth degree B-spline is completed which is an approximate solution of the problem given by Eqs. 1 and 2.

\section{Error bound}

In this section, an error bound of the fourth degree $\mathrm{B}$-spline is formulated Denote Eq.11 by $A C=Y$, and Let $\hat{\mathrm{S}}(x)=\sum_{j=i-2}^{i+2} \hat{\mathrm{C}}_{j} B_{j}(x)$ be an approximate solution of $\hat{\mathrm{Y}}$, and $\hat{\mathrm{Y}}$ be an approximate solution of $Y$. 


\section{Lemma 1}

If $A$ is an $n \times n$ matrix as defined in Eq.11, then $\left\|A^{-1}\right\|_{\infty}=\frac{1}{24}$

\section{Proof:}

It easy to show that by mathematical induction the matrix $\mathrm{A}$ is invertible, then It is known that $\|A\|=\max \|A x\|$, or $\|A\|_{\infty}=\max _{j} \sum_{i=1}^{n}|a i j|$, then

$$
\begin{aligned}
& \left\|A^{-1}\right\|=\max _{\|x\|=1}\left\|A^{-1} x\right\|=\max _{\|A y\|=1}\|y\|=\left(\min _{\|A y\|=1}\|y\|^{-1}\right)^{-1}=(\min \|A x\|)^{-1}, \\
& \quad \text { where } A^{-1} x=y \\
& =(\min (24,32,48))^{-1}=\frac{1}{24}
\end{aligned}
$$

\section{Lemma 2}

Let $\hat{S}(x)=\sum_{j=i-2}^{i+2} \hat{\mathrm{C}}_{j} B_{j}(x)$ be another approximate solution of $\hat{Y}$ using exact boundary conditions, then

$\|\hat{\mathrm{C}}-C\|_{\infty} \leq \frac{1}{24}\|Y-\hat{\mathrm{Y}}\|_{\infty}$,

\section{Proof:}

For the approximate solution $\hat{S}(x)$ of $\hat{Y}$, another matrix system could be obtained as follows;

$$
A \hat{\mathrm{C}}=\hat{\mathrm{Y}} \text {, and }
$$

$A C=Y \rightarrow A C-A \hat{\mathrm{C}}=Y-\hat{\mathrm{Y}}$,

Then $A(C-\hat{\mathrm{C}})=(Y-\hat{\mathrm{Y}}) \rightarrow(C-\hat{\mathrm{C}})=A^{-1}(Y-\hat{\mathrm{Y}})$

Now using properties of norm, the following yields;

$$
\|\hat{\mathrm{C}}-C\|_{\infty}=\left\|A^{-1}(Y-\hat{\mathrm{Y}})\right\|_{\infty} \leq\left\|A^{-1}\right\|_{\infty}\|Y-\hat{\mathrm{Y}}\|_{\infty} \leq \frac{1}{24}\|Y-\hat{\mathrm{Y}}\|_{\infty}
$$

\section{Lemma 3}

The following inequalities are true for $i=0,1,2, \ldots, n$

i) $\sum_{i=-2}^{n+1}\left|B_{i}(x)\right|=24$,

ii) $\sum_{i=-2}^{n+1}\left|B_{i}^{\prime}(x)\right| \leq \frac{32}{h}$,

iii) $\sum_{i=-2}^{n+1}\left|B_{i}^{\prime \prime}(x)\right| \leq \frac{48}{h^{2}}, \quad$ and

\section{Proof}

iv) $\sum_{i=-2}^{n+1}\left|B_{i}^{\prime \prime \prime}(x)\right| \leq \frac{192}{h^{3}}$,

For $x \in\left[x_{i}, x_{i+1}\right]$, and from Table 1 , for $i=0,1,2, \ldots, n$

i) $\quad \sum_{i=-2}^{n+1}\left|B_{i}(x)\right|=\sum_{i=-2}^{n+1} B_{i}(x)=1+11+11+1=24$,

ii) $\quad \sum_{i=-2}^{n+1}\left|B_{i}{ }^{\prime}(x)\right| \leq \frac{4}{h}+\frac{12}{h}+\frac{12}{h}+\frac{4}{h} \leq \frac{32}{h}$,

iii) $\quad \sum_{i=-2}^{n+1}\left|B_{i}^{\prime \prime}(x)\right| \leq \frac{48}{h^{2}}$,

iv) $\quad \sum_{i=-2}^{n=i+1}\left|B_{i}{ }^{\prime \prime \prime}(x)\right| \leq \frac{192}{h^{3}}$.

\section{Lemma 4}

Let $S(x)$ be a quartic B-spline approximate solution of $y(x)$ with lacunary and approximate boundary conditions, and let $\hat{S}(\mathrm{x})$ be another approximate solution of $y(x)$ with lacunary boundary conditions, then for $i=0,1,2, \ldots, n$, the following are true 
i) $\quad|S(x)-\hat{\mathrm{S}}(\mathrm{x})| \leq \frac{1}{24}\|Y-\hat{\mathrm{Y}}\|_{\infty}$,

ii) $\quad\left|S^{\prime}(x)-\hat{S}^{\prime}(\mathrm{x})\right| \leq \frac{4}{3 h}\|Y-\hat{\mathrm{Y}}\|_{\infty}$,

iii) $\left|S^{\prime \prime}(x)-\hat{S}^{\prime \prime}(\mathrm{x})\right| \leq \frac{2}{h^{2}}\|Y-\hat{\mathrm{Y}}\|_{\infty}$,

iv) $\quad\left|S^{\prime \prime \prime}(x)-\hat{S}^{\prime \prime \prime}(\mathrm{x})\right| \leq \frac{8}{h^{3}}\|Y-\hat{\mathrm{Y}}\|_{\infty}$.

\section{Proof}

Using Lemma 2 and Lemma 3, and for $k=0,1,2,3$, we have

$\left|S^{(k)}(x)-\hat{\mathrm{S}}^{(\mathrm{k})}(\mathrm{x})\right|=\left|\sum_{i=-2}^{n+1}\left(C_{i}-\hat{\mathrm{C}}_{i}\right) B_{i}^{(k)}(x)\right| \leq\|C-\hat{\mathrm{C}}\|_{\infty} \sum_{i=-2}^{n+1}\left|B_{i}^{(k)}(x)\right|$

i) $\quad$ For $k=0$,

$|S(x)-\hat{\mathrm{S}}(\mathrm{x})| \leq\|C-\hat{\mathrm{C}}\|_{\infty} \sum_{i=-2}^{i=n+1}\left|B_{i}(x)\right| \leq\|Y-\hat{\mathrm{Y}}\|_{\infty}$.

ii) $\quad$ For $k=1$,

$\left|S^{\prime}(x)-\hat{S}^{\prime}(\mathrm{x})\right| \leq\|C-\hat{\mathrm{C}}\|_{\infty} \sum_{i=-2}^{n+1}\left|B_{i}^{\prime}(x)\right| \leq \frac{4}{3 h}\|Y-\hat{\mathrm{Y}}\|_{\infty}$.

iii) $\quad$ For $k=2$;

$\left|S^{\prime \prime}(x)-\hat{S}^{\prime \prime}(\mathrm{x})\right| \leq\|C-\hat{\mathrm{C}}\|_{\infty} \sum_{i=-2}^{i=n+1}\left|B_{i}^{\prime \prime}(x)\right| \leq \frac{2}{h^{2}}\|Y-\hat{\mathrm{Y}}\|_{\infty}$.

iv) Finally for $k=3$;

$$
\left|S^{\prime \prime \prime}(x)-\hat{\mathrm{S}}^{\prime \prime \prime}(\mathrm{x})\right| \leq\|C-\hat{\mathrm{C}}\|_{\infty} \sum_{i=-2}^{n+1}\left|B_{i}^{\prime \prime \prime}(x)\right| \leq \frac{8}{h^{3}}\|Y-\hat{\mathrm{Y}}\|_{\infty} .
$$

For $f \in C^{4}[0,1]$, we have the following expansions, that will be used in subsequent section.

$$
\begin{aligned}
& \hat{\mathrm{S}}\left(x_{i}\right)=y\left(x_{i}\right)+2 h y^{\prime}\left(x_{i}\right)+2 h^{2} y^{\prime \prime}\left(x_{i}\right)+\frac{4}{3} h^{3} y_{i}^{\prime \prime \prime}\left(x_{i}\right)+\frac{2}{3} h^{4} y_{i}^{(4)}\left(\theta_{1, i}\right) \\
& \hat{\mathrm{S}}^{\prime}\left(x_{i}\right)=y^{\prime}\left(x_{i}\right)+2 h y^{\prime \prime}\left(x_{i}\right)+\frac{2}{3} h^{2} y_{i}^{\prime \prime \prime}\left(x_{i}\right)+\frac{2}{9} h^{3} y_{i}^{(4)}\left(\theta_{2, i}\right) \\
& \hat{\mathrm{S}}^{\prime \prime}\left(x_{i}\right)=y^{\prime \prime}\left(x_{i}\right)+\frac{2}{3} h y_{i}^{\prime \prime \prime}\left(x_{i}\right)+\frac{2}{9} h^{2} y_{i}^{(4)}\left(\theta_{3, i}\right) \\
& \hat{\mathrm{S}}^{\prime \prime \prime}\left(x_{i}\right)=y^{\prime \prime \prime}\left(x_{i}\right)+\frac{2}{3} h y_{i}^{(4)}\left(\theta_{4, i}\right)
\end{aligned}
$$

where $\theta_{j, i} \in\left[x_{0}, x_{n}\right], j=1,2,3,4$

\section{Theorem 1}

Let $S(x)$ be a fourth degree B-Spline approximation solution of $Y$, then the following inequalities are true;

i) $\quad\left|S^{\prime}(x)-Y^{\prime}(\mathrm{x})\right| \leq \frac{4}{3 h}\|Y-\hat{\mathrm{Y}}\|_{\infty}+2 h\left\|y^{\prime \prime}\left(x_{i}\right)\right\|+\frac{2}{3} h^{2}\left\|y_{i}^{\prime \prime \prime}\left(x_{i}\right)\right\|+\frac{2}{9} h^{3} w\left(y_{i}^{(4)}\left(\theta_{2, i}\right)\right)$

ii) $\quad\left|S^{\prime \prime}(x)-Y^{\prime \prime}(\mathrm{x})\right| \leq \frac{2}{h^{2}}\|Y-\hat{\mathrm{Y}}\|_{\infty}+\frac{2}{3} h\left\|y_{i}^{\prime \prime \prime}\left(x_{i}\right)\right\|+\frac{2}{9} h^{2} w\left(y^{(4)}\left(\theta_{3, i}\right)\right)$

iii) $\quad\left|S^{\prime \prime \prime}(x)-Y^{\prime \prime \prime}(\mathrm{x})\right| \leq \frac{8}{h^{3}}\|Y-\hat{\mathrm{Y}}\|_{\infty}+\frac{2}{3} h w\left(f^{(4)}\left(\theta_{3, i}\right)\right)$

\section{Proof:}

Using lemma 4, prove of (iv) is as follows;

$\left|S^{\prime \prime \prime}(x)-Y^{\prime \prime \prime}(\mathrm{x})\right| \leq\left|S^{\prime \prime \prime}(x)-\hat{\mathrm{S}}^{\prime \prime \prime}(\mathrm{x})\right|+\left|\hat{\mathrm{S}}^{\prime \prime \prime}(x)-Y^{\prime \prime \prime}(\mathrm{x})\right| \leq \frac{8}{h^{3}}\|Y-\hat{\mathrm{Y}}\|_{\infty}+\left|\hat{\mathrm{S}}^{\prime \prime \prime}(x)-Y^{\prime \prime \prime}(\mathrm{x})\right|$ Assigning $\hat{\mathrm{S}}^{\prime \prime \prime}(x)$ as $\hat{\mathrm{S}}^{\prime \prime \prime}{ }_{i}(x)$ and $Y^{\prime \prime \prime}(\mathrm{x})$ as ${y_{i}}^{\prime \prime \prime}\left(x_{i}\right)$, and using Eq. 12 , then the last term of above equation can be obtained as follows;

$\left|\hat{S}^{\prime \prime \prime}\left(x_{i}\right)-Y^{\prime \prime \prime}\left(x_{i}\right)\right|=\left|y^{\prime \prime \prime}\left(x_{i}\right)+\frac{2}{3} h y_{i}^{(4)}\left(\theta_{1, i}\right)-y^{\prime \prime \prime}\left(x_{i}\right)\right|=\left|\frac{2}{3} h y^{(4)}\left(\theta_{4, i}\right)\right|$ 
Hence

$\left|S^{\prime \prime \prime}(x)-Y^{\prime \prime \prime}(\mathrm{x})\right| \leq \frac{8}{h^{3}}\|Y-\hat{\mathrm{Y}}\|_{\infty}+\frac{2}{3} h w\left(y^{(4)}\left(\theta_{4, i}\right)\right)$, where $i=0,1,2, \ldots, n$.

Similar to the proof of (iv) the followings can be proved;

iii) $\quad\left|S^{\prime \prime}(x)-Y^{\prime \prime}(\mathrm{x})\right| \leq\left|S^{\prime \prime}(x)-\hat{S}^{\prime \prime}(\mathrm{x})\right|+\left|\hat{S}^{\prime \prime}(x)-Y^{\prime \prime}(\mathrm{x})\right| \leq \frac{2}{h^{2}}\|Y-\hat{\mathrm{Y}}\|_{\infty}+\left|\hat{S}^{\prime \prime}(x)-Y^{\prime \prime}(\mathrm{x})\right|$

Assigning $\hat{\mathrm{S}}^{\prime \prime}(x)$ as $\hat{\mathrm{S}}^{\prime \prime}{ }_{i}(x)$ and $Y^{\prime \prime}(\mathrm{x})$ as $y_{i}{ }^{\prime \prime}\left(x_{i}\right)$, then the last term of the above equation can be obtained as follows;

Hence

$$
\begin{gathered}
\left|\hat{\mathrm{S}}^{\prime \prime}\left(x_{i}\right)-Y^{\prime \prime}\left(\mathrm{x}_{\mathrm{i}}\right)\right|=\left|y^{\prime \prime}\left(x_{i}\right)+\frac{2}{3} h y_{i}^{\prime \prime \prime}\left(x_{i}\right)+\frac{2}{9} h^{2} y_{i}^{(4)}\left(\theta_{3, i}\right)-y^{\prime \prime}\left(x_{i}\right)\right| \\
=\left|\frac{2}{3} h y_{i}^{\prime \prime \prime}\left(x_{i}\right)+\frac{2}{9} h^{2} y_{i}^{(4)}\left(\theta_{3, i}\right)\right| \\
=\frac{2}{3} h\left\|y_{i}^{\prime \prime \prime}\left(x_{i}\right)\right\|+\frac{2}{9} h^{2} w\left(y^{(4)}\left(\theta_{3, i}\right)\right)
\end{gathered}
$$

$\left|S^{\prime \prime}(x)-Y^{\prime \prime}(\mathrm{x})\right| \leq \frac{2}{h^{2}}\|Y-\hat{\mathrm{Y}}\|_{\infty}+\frac{2}{3} h\left\|y^{\prime \prime \prime}\left(x_{i}\right)\right\|_{\infty}+\frac{2}{9} h^{2} w\left(y^{(4)}\left(\theta_{3, i}\right)\right)$ where $i=0,1,2, \ldots, n$.

Applying similar techniques complete the proof of the theorem

ii) $\quad\left|S^{\prime}(x)-Y^{\prime}(\mathrm{x})\right| \leq\left|S^{\prime}(x)-\hat{\mathrm{S}}^{\prime}(x)\right|+\left|\hat{\mathrm{S}}^{\prime}(x)-Y^{\prime}(\mathrm{x})\right|$

$$
\begin{aligned}
& \leq \frac{4}{3 h}\|Y-\hat{\mathrm{Y}}\|_{\infty}+\left|\hat{\mathrm{S}}^{\prime}(x)-Y^{\prime}(x)\right| \\
& \leq \frac{4}{3 h}\|Y-\hat{\mathrm{Y}}\|_{\infty}+\left|\hat{\mathrm{S}}^{\prime}\left(x_{i}\right)-Y^{\prime}\left(x_{i}\right)\right| \\
& \leq \frac{4}{3 h}\|Y-\hat{\mathrm{Y}}\|_{\infty}+\left|y^{\prime}\left(x_{i}\right)+2 h y^{\prime \prime}\left(x_{i}\right)+\frac{2}{3} h^{2} y_{i}^{\prime \prime \prime}\left(x_{i}\right)+\frac{2}{9} h^{3} y_{i}^{(4)}\left(\theta_{2, i}\right)-y^{\prime}\left(x_{i}\right)\right|
\end{aligned}
$$$$
\leq \frac{4}{3 h}\|Y-\hat{Y}\|_{\infty}+\left|2 h y^{\prime \prime}\left(x_{i}\right)+\frac{2}{3} h^{2} y_{i}^{\prime \prime \prime}\left(x_{i}\right)+\frac{2}{9} h^{3} y_{i}^{(4)}\left(\theta_{2, i}\right)\right|
$$$$
\leq \frac{4}{3 h}\|Y-\hat{Y}\|_{\infty}+2 h\left\|y^{\prime \prime}\left(x_{i}\right)\right\|+\frac{2}{3} h^{2}\left\|y_{i}^{\prime \prime \prime}\left(x_{i}\right)\right\|+\frac{2}{9} h^{3} w\left(y_{i}^{(4)}\left(\theta_{2, i}\right)\right)
$$

i) $\quad|S(x)-Y(\mathrm{x})| \leq|S(x)-\hat{\mathrm{S}}(\mathrm{x})|+|\hat{\mathrm{S}}(x)-Y(\mathrm{x})|$

$$
\leq\|Y-\hat{\mathrm{Y}}\|_{\infty}+\left|\hat{\mathrm{S}}\left(x_{i}\right)-Y\left(x_{i}\right)\right|
$$

$\leq\|Y-\hat{Y}\|_{\infty}+\left|y\left(x_{i}\right)+2 h y^{\prime}\left(x_{i}\right)+2 h^{2} y^{\prime \prime}\left(x_{i}\right)+\frac{4}{3} h^{3} y_{i}^{\prime \prime \prime}\left(x_{i}\right)+\frac{2}{3} h^{4} y_{i}^{(4)}\left(\theta_{1, i}\right)-y\left(x_{i}\right)\right| \leq$ $\|Y-\hat{Y}\|_{\infty}+2 h\left\|y^{\prime}\left(x_{i}\right)\right\|+2 h^{2}\left\|y_{i}^{\prime \prime}\left(x_{i}\right)\right\|+\frac{4}{3} h^{3}\left\|y_{i}^{\prime \prime \prime}\left(x_{i}\right)\right\|+w\left(y_{i}^{(4)}\left(\theta_{1, i}\right)\right)$

\section{Example 1:}

$y^{(4)}(x)+x y=-\left(8+7 x+x^{3}\right) e^{x}$ with

$y^{\prime}(0)=1$

$y^{\prime}(1)=-e$

$y^{\prime \prime}(0)=0$

The analytical solution is given by $y(x)=x(1-x) e^{x}$

Table 2 compares the numerical results between present B-Spline method and the computational method used in [12] 
Table 2

\begin{tabular}{|l|l|l|l|}
\hline$x_{i}$ & Exact Solution & {$[12]$} & B-Spline Max Error \\
\hline $1 / 8$ & -0.1239381121 & $2.37 e^{-8}$ & $8.94 e^{-9}$ \\
\hline $1 / 16$ & -0.0437870146 & $5.75 e^{-9}$ & $3.46 e^{-9}$ \\
\hline $1 / 32$ & -0.0312344196 & $1.47 e^{-9}$ & $5.59 e^{-10}$ \\
\hline
\end{tabular}

Error of the present B-spline method is slightly better than the error obtained in [12], this confirms that the BSpline method is a precise one for the models of BVPs stated in section 2.

\section{Example 2:}

$y^{(4)}(x)+4 y(x)=1$, with

$y^{\prime}(0)=0$

$y^{\prime}(1)=\frac{0.664 \sin 1 \sinh 1-1.932 \cos 1 \cosh 1}{2(\cosh 2+\cos 2)}$

$y^{\prime \prime}(0)=-\frac{\sin 1 \sinh 1}{\cosh 2+\cos 2}$

The analytical solution of above problem is;

$y(x)=0.25\left[1-2 \frac{\sin 1 \sinh 1 \sin x \sinh x+\cos 1 \cosh 1 \cos x \cosh x}{\cosh 2+\cos 2}\right.$

The results of maximum absolute error $\max \left[y^{(r)}\left(x_{i}\right)\right]=\max _{1 \leq i \leq n}\left|y^{(r)}\left(x_{i}\right)-S^{(r)}\left(x_{i}\right)\right|, r=0,1,2,3$ are tabulated in Table 3

Table 3

\begin{tabular}{|l|l|l|l|}
\hline$x_{i}$ & Exact Solution & {$[12]$} & B-spline Error \\
\hline $1 / 8$ & 0.07372 & $1.29 e^{-7}$ & $7.53 e^{-8}$ \\
\hline $1 / 16$ & 0.08767 & $3.08 e^{-8}$ & $4.21 e^{-9}$ \\
\hline $1 / 32$ & 0.08858 & $7.54 e^{-9}$ & $3.17 e^{-9}$ \\
\hline
\end{tabular}

The example has been solved by Yogesh and Punkja. [12], The numerical results shown in Table 3 shows encouraging results of our method.

\section{Conclusion}

Based on the investigation done in the present study, it can be concluded that, B-Spline is fast, flexible and precise to be used to find approximate solutions of boundary value problems with given limited lacunary interpolation condition and approximate boundary condition, below are some conclusions;

1) B-Spline produced an approximation of analytical solution of the problem with respect to the selected subinterval.

2) B-Spline is a good tool to be used to solve lacunary interpolation problems for Boundary Value Problems.

3) It is considerable that as the subintervals are increasing, and $h$ being small, the approximation by B-Spline is more precise and has potential to give good approximation solution for boundary value problems.

4) B-Spline of degree four is precise and flexible enough to become an approximate solution of BVPs. 
5) The errors in the numerical examples are quite good, slightly better than other errors obtained from other approximation tools.

\section{References}

[1] S. Shafie and A. A. Majid, Approximation of cubic B- spline interpolation method, shooting and finite difference methods for linear problems on solving linear two-point boundary value. 2012.

[2] R. K. Saeed, K. H. Jwamer, and F. K. Hamasalh, Introduction to numerical analysis, University of Sulaimani, Sulaimani, Kurdistan Region- Iraq. 2015.

[3] V. Dahiya, Exploring B-spline functions for numerical solution of mathematical problems, Int. J. of Multidisciplinary Research and Development, 2(1) 2015, : 452-458

[4] P. J. Davis, Interpolation and Approximation, Blaisdell, New York. 1961.

[5] C. De-Boor, A practical guide to splines, Springer-Verlag, Berlin. 1978.

[6] Y. P. Dubey, Best error bounds for splines of degree six, Int. Journal of Math. Analysis, 5(24) 2011: 1201-1209.

[7] Y. P. Dubeyand A. Shukla, The deficient $C^{1}$ quartic spline interpolation, Research Inventy, Int. J. of Engineering and Sciences, 2(9) 2013, 24-30.

[8] C. A. Hall and W. W. Meyer, Optimal error bounds for cubic spline interpolation, J. Approx. Theory, 16: (1976) 105-122.

[9] K. H. Jwamer, Minimizing error bounds in $(0,2,3)$ lacunary interpolation by sextic spline function, Journal of Mathematics and Statistics, 3(4) 2007,: 249-256.

[10] S. S. Rana and Y. P. Dubey, Best error bounds of deficient quantic spline interpolation, Indian J. Pure Appl. Math., 28(10) 1997, 1337-1344.

[11] Saeed, R.K. (1990), A study of lacunary interpolation by splines, MSC Thesis, Salahaddin University/Erbil, Iraq.

[12] Yogesh Gupta et al, A Computational Method for Solving Two Point Boundary Value Problems of Order Four. Int. J. Comp. Tech. Appl, 2 (5) 2011, 1426-1431 